\title{
Using SOMs to Gain Insight into Human Language Processing
}

\author{
Risto Miikkulainen
}

\begin{abstract}
While SOMs are commonly used as a tool for data visualization and data analysis, they can also serve as a model for cognitive functions in humans. Such functions include semantic and episodic memory, vision, and language. In this talk I will review how elements of sentence meaning can be laid out on a map, resulting in human-like graded semantic understanding instead of a single parse tree. I will also describe a model of the lexicon that can be fit to the individual patient with aphasia, and used to predict optical rehabilitation treatments.
\end{abstract}

Keywords Self-organizing maps - Cognitive models $\cdot$ Semantic understanding Lexicon model 